\title{
Development of Ring-imaging Cherenkov Counter for Heavy lons
}

M. Machida*

Department of Physics, Tokyo University of Science, Noda, Chiba 278-8510, Japan

E-mail: 6216625ded.tus.ac.jp

J. Chiba ${ }^{1}$, D. Nishimura ${ }^{1}$, M. Fukuda ${ }^{2}$, H. Du${ }^{2}$, S. Yagi ${ }^{2}$, S. Nakamura ${ }^{2}$, S. Fukuda ${ }^{4}$, A. Kitagawa $^{4}$, S. Sato ${ }^{4}$, M. Mihara ${ }^{2}$, M. Tanaka ${ }^{2}$, K. Ohnishi $^{2}$, T. Suzuki ${ }^{6}$, T. Ohtsubo ${ }^{3}$, M. Takechi' ${ }^{3}$, T. Izumikawa ${ }^{5}$, A. Homma ${ }^{3}$, E. Miyata ${ }^{3}$, A. Ikeda ${ }^{3}$, N. Kanda ${ }^{3}$, T. Yamaguchi $^{6}$, I. Kato ${ }^{6}$, H. Oikawa ${ }^{1}$, H. Hosokawa ${ }^{1}$, J. Ohnishi ${ }^{1}$, M. Tada $^{1}$, R. Ishii ${ }^{1}$, T. Tahara $^{1}$ T. Moriguchi ${ }^{7}$, M. Amano ${ }^{7}$

${ }^{1}$ Department of Physics, Tokyo University of Science, Noda, Chiba 278-8510, Japan

${ }^{2}$ Department of Physics, Osaka University, Toyonaka, Osaka 560-0043, Japan

${ }^{3}$ Department of Physics, Niigata University, Niigata 950-2102, Japan

${ }^{4}$ National Institute of Radiological Sciences, Chiba 263-8555, Japan

${ }^{5}$ RI Center, Niigata University, Niigata 950-8510, Japan

${ }^{6}$ Department of Physics, Saitama University, Saitama 338-8570, Japan

${ }^{7}$ Department of Physics, University of Tsukuba, Ibaraki 305-8571, Japan

Up to this day, RICHes have been mainly used for high energy experiments to identify leptons and mesons. On the other hand, the number of Cherenkov photons is proportional to square of the atomic number, so that application of a RICH for heavy ions is expected. Then we are developing a ring-imaging Cherenkov counter for heavy ions (HI-RICH). The experiment was performed at NIRS-HIMAC. As for the primary beam $\left({ }^{132} \mathrm{Xe}\right.$ with an energy of $\left.420 \mathrm{MeV} / \mathrm{u}\right)$, a velocity resolution $\Delta \beta / \beta=0.050 \%$ (standard deviation $\sigma$ ) and a detection efficiency $\varepsilon=99.9 \%$ were achieved. Regarding to the secondary beam, a particle identification was successfully performed with HI-RICH and an ion chamber.

The 26th International Nuclear Physics Conference

11-16 September, 2016

Adelaide, Australia

${ }^{*}$ Speaker. 


\section{Introduction}

The ring-imaging Cherenkov counter (RICH) is a velocity detector for incident charged particles. RICHes have been mainly developed and used for experimental particle physics. For example, the first RICH tested at CERN [W] was performed with ionization process and the Aerogel RICH counter in the KEK Belle II experiment [[] which is going to start in 2017 will be used to separate kaons from pions and to provide discrimination between pions, muons and electrons. In nuclear physics experiments, on the other hand, GSI developed a RICH for heavy ions with a liquid $\mathrm{C}_{6} \mathrm{~F}_{14}$ radiator and a MWPC as a photon detector in 1999 [B]], but this is a rare case. A velocity resolution of this was measured to be $\Delta \beta / \beta=0.03 \%(\sigma)$ for $\mathrm{Ru}^{44+}$ particles of $\beta \simeq 0.85$.

Also we have developed a RICH for heavy ions with several hundreds MeV/u energy (HI$\mathrm{RICH}$ ). The conventional velocity measurement for heavy ions is time-of-flight method (TOF), which requires large space and high costs for high accuracy. In contrast, HI-RICH requires only decades of centimeters, so that this is expected to be a new compact and low-cost velocity detector.

The velocity $\beta$ of ions can be deduced by measuring the Cherenekov angles $\theta_{\mathrm{C}}\left(\cos \theta_{\mathrm{C}}=\right.$ $(n \beta)^{-1}$, where $n$ is the refractive index of a radiator). For measuring $\theta_{\mathrm{C}}$, bottom face rings of the Cherenkov cones should be reconstructed. Note that this angular distribution spreads mainly due to energy loss of incident particles in a radiator, optical dispersion $n(\lambda)$ of emitted radiation and finite thickness of a radiator. The number of photons emitted per unit wavelength $\lambda$ per unit length of radiator $x$ is then,

$$
\frac{\mathrm{d} N}{\mathrm{~d} \lambda \mathrm{d} x}=\frac{2 \pi \alpha Z^{2}}{\lambda^{2}}\left(1-\frac{1}{n^{2} \beta^{2}}\right)
$$

where $\alpha$ is the fine structure constant and $Z$ is the atomic number of the incident particle.

\section{Experiment}

\subsection{Beam line}

The experiment was performed at NIRS-HIMAC, with medical accelerator for Heavy ions. Several detectors including HI-RICH were placed on the last focal plane (F3) in the SB2 course which is shown in Ref. [四]. The primary beam was ${ }^{132} \mathrm{Xe}$ with a kinetic energy of $420 \mathrm{MeV} / \mathrm{u}$ which is equivalent to a velocity $\beta=0.725$. As for the secondary beam experiment, the production target was a $2 \mathrm{~mm}$-thick beryllium.

\subsection{Detector setup}

Figure $\square$ shows a top view of the detector setup. The size of the plastic scintillator was $50 \mathrm{~mm} \times$ $50 \mathrm{~mm} \times 1 \mathrm{mmt}$, whose signals were read by coincidences of two PMTs. It was used as a common trigger. The ion chamber is an energy loss $\Delta E$ detector which was used for particle identifications.

\subsection{HI-RICH design}

Figure $\square$ shows a schematic view of HI-RICH, which is consist of a radiator and three pairs of $8 \times 8$ multi-anode photomultiplier tubes which are placed perpendicular to the beam line. The PMTs are covered with band-pass filters with wavelength peaks of $360 \mathrm{~nm}$. Radiators can be moved along the beam line. 


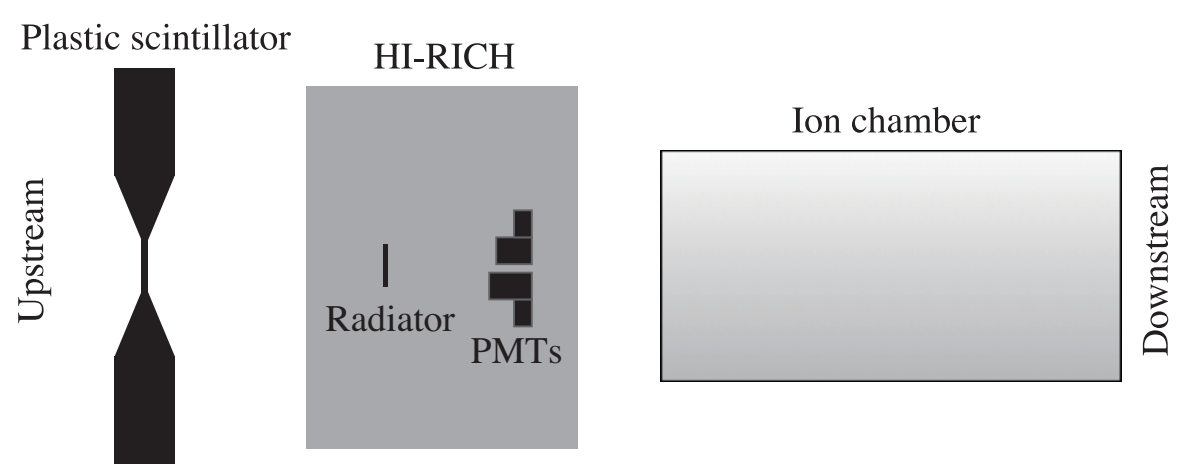

Figure 1: Top view of the detector setup

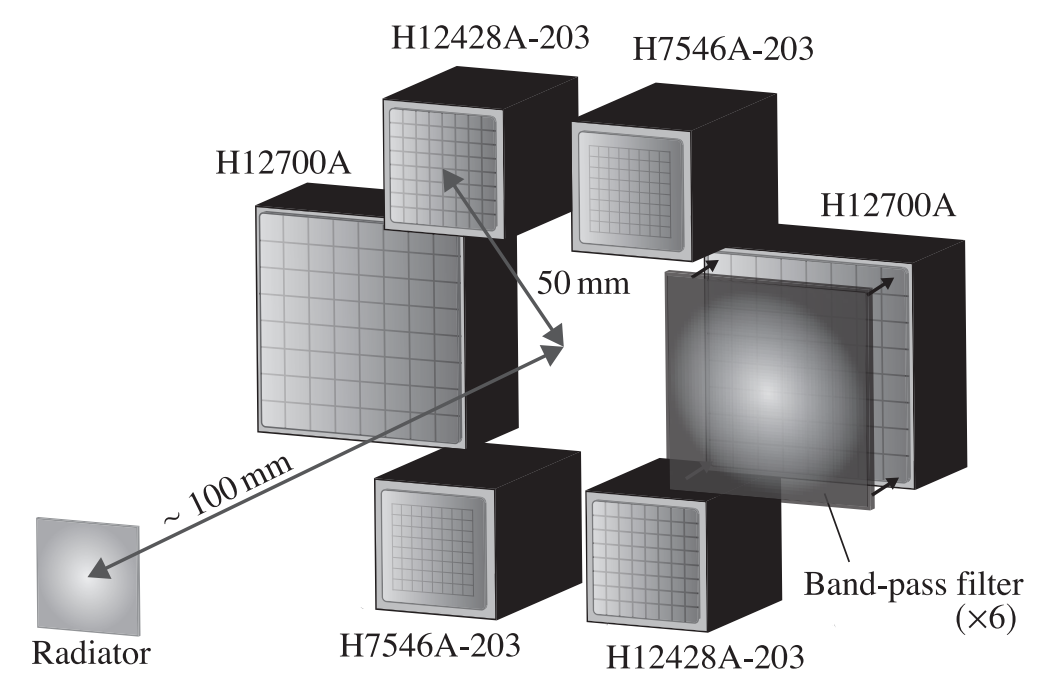

Figure 2: Schematic view of HI-RICH

\subsection{Photomultiplier tubes}

The model number of the PMTs are H7546A-203, H12428A-203 and H12700A which are made by Hamamatsu Photonics. As shown in Fig. వ, they are $8 \times 8$ multi-anode models. The maximum quantum efficiencies of the PMTs are about $30-40 \%$ and the peak wavelengths of them are $350 \mathrm{~nm}$.

\subsection{Radiator}

As for radiators, synthetic quartz $\left(\mathrm{SiO}_{2}\right)$ and $\mathrm{BK} 7$ were used. Refractive indexes of $\mathrm{SiO}_{2}$ and BK7 are about 1.48 and 1.54 respectively. Each optical dispersion $n(\lambda)$ is as follows:

$$
\begin{aligned}
& n_{\text {quartz }}(\lambda)=1.45+0.24 \exp [-0.0064 \lambda]+18.93 \exp [-0.032 \lambda] \\
& n_{\mathrm{BK} 7}(\lambda)=1.51+0.1 \exp [-0.00566 \lambda]+0.213 \exp [-0.0078 \lambda]
\end{aligned}
$$

A graph of them in a range of 300-400 nm are shown in Fig. [3. A red line and a blue dashed line show $\mathrm{BK} 7$ and $\mathrm{SiO}_{2}$ respectively. 


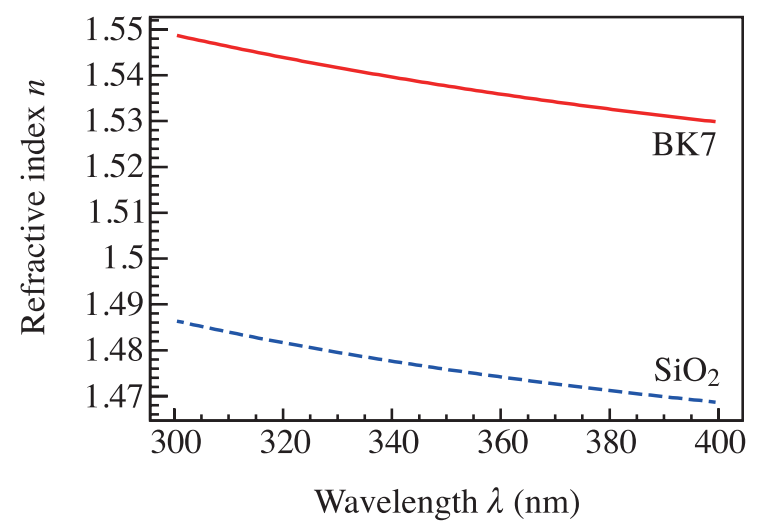

Figure 3: Optical dispersion of radiators

\subsection{Filter}

There are band-pass filters with a thickness of $2.5 \mathrm{~mm}$. As shown in Fig. 乙, The PMTs are covered with them. The model number is $U V U-360$ made by Edmund Optics. They suppress spreads of angles of emitted photons by optical dispersion. In the wavelength dependence of the transmission, the peak wavelength is $360 \mathrm{~nm}$ and the FWHM is $45 \mathrm{~nm}$. A graph of this is shown in Ref. [5]].

\section{Results and discussion}

As an example, 2D plots of detected Cherenkov photons of all events seen from upstream are shown in Fig. 团. This is a result of the primary beam with a $\mathrm{SiO}_{2}$ radiator with a thickness of $0.48 \mathrm{~mm}$. Figure [ s shows a time spectrum of a channel. The time information is differences between signals of the HI-RICH PMTs and common trigger signals of the plastic scintillator. The FWHM of the spectrum is $0.38 \mathrm{~ns}$, which is consistent with the catalog value of a transit time spread of $0.35 \mathrm{~ns}$.

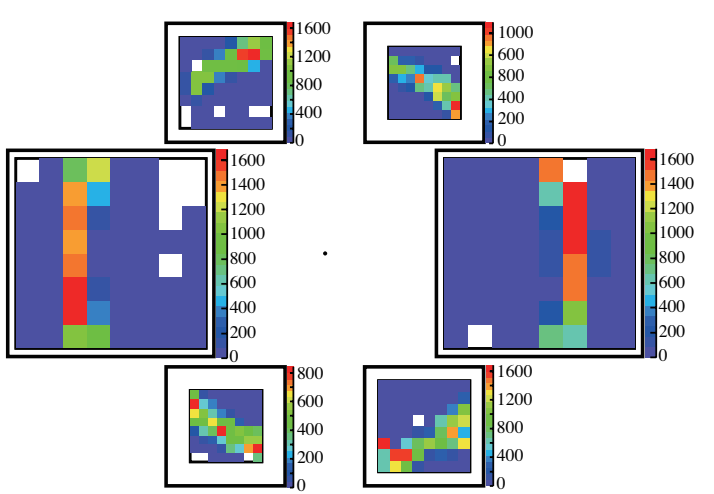

Figure 4: Detected Cherenkov photons (all events)

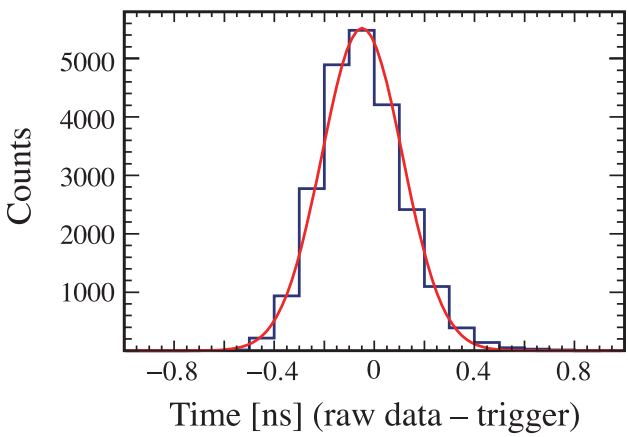

Figure 5: Time spectrum of a HI-RICH channel 


\subsection{Monte Carlo simulation}

In order to understand the property of HI-RICH, We simulate HI-RICH. Since results will be compared with Monte Carlo simulations in this paper, we explain the methods of the simulations briefly.

Fluctuations taken into consideration are as follows:

- Energy loss of beams in a radiator

This is the most dominant fluctuation and was calculated with LISE++.

- Angles of emitted photons by optical dispersion

A spread of wavelength was considered as a rectangular distribution. The center and the full width of it was $360 \mathrm{~nm}$ and $45 \mathrm{~nm}$ which is approximation of the wavelength dependence of

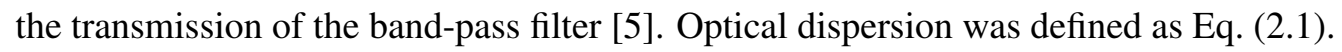

- Positions of emitted photons in a radiator

Because of radiator thickness, positions of emitted photons is uncertain.

The number of photoelectrons was considered as a Poisson distribution. If a photoelectron reached a position of a PMT channel, the channel was considered to be hit. This process was repeated with all photoelectrons.

\subsection{Primary beam}

The primary beam was ${ }^{132} \mathrm{Xe}$ with an energy of $420 \mathrm{MeV} / \mathrm{u}$ and the estimated velocity just before HI-RICH was $\beta=0.712$.

Table $\mathbb{W}$ shows experimental results of the velocity resolutions and detection efficiencies about two kinds of thicknesses of $\mathrm{SiO}_{2}$ radiators. The experimental velocity resolutions are compared with the simulation in Fig. 6. The simulated detection efficiencies are $100 \%$. If a radiator is too thick, velocity resolution will be large primarily because of energy loss in it. If a radiator is too thin, however, velocity resolution will be large too owing to lack of the number of photons. Data points and a blue line are experimental and simulation results respectively. The experimental data of the velocity resolutions are not achieved to the simulation. This is because the real number of photoelectrons is fewer than the theoretical number of them owing to discriminating signals. Because the number of photons is approximately proportional to the radiator thickness, so is the number of photoelectrons. Then a red dashed line shows simulation results performed as the actual number of photoelectrons. These are consistent with experimental results. A velocity spectrum with a radiator thickness of $0.95 \mathrm{~mm}$ is shown in Fig. .

Table 1: Experimental results of $\beta$ resolution and the detection efficiency

\begin{tabular}{ccc}
\hline $\mathrm{SiO}_{2}$ radiator thickness & $0.48 \mathrm{~mm}$ & $0.95 \mathrm{~mm}$ \\
\hline$\Delta \beta / \beta(\%)(\sigma)$ & $0.0547(3)$ & $0.0497(3)$ \\
Detection efficiency $\varepsilon(\%)$ & $99.39(6)$ & $99.86(3)$ \\
\hline
\end{tabular}




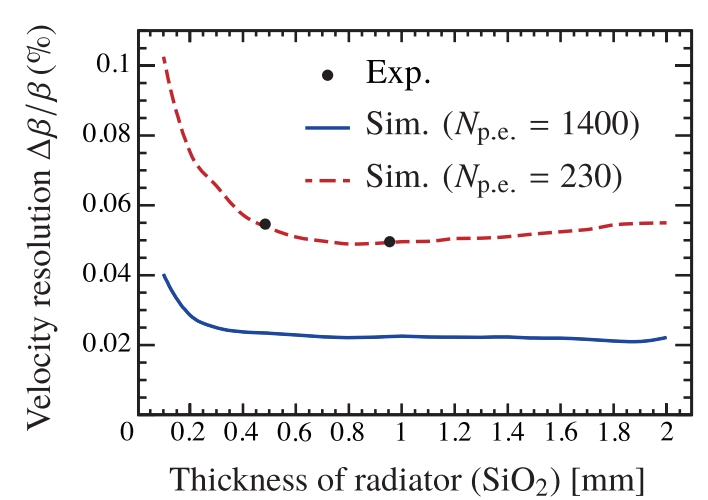

Figure 6: $\mathrm{SiO}_{2}$ radiator thickness dependence of the velocity resolution

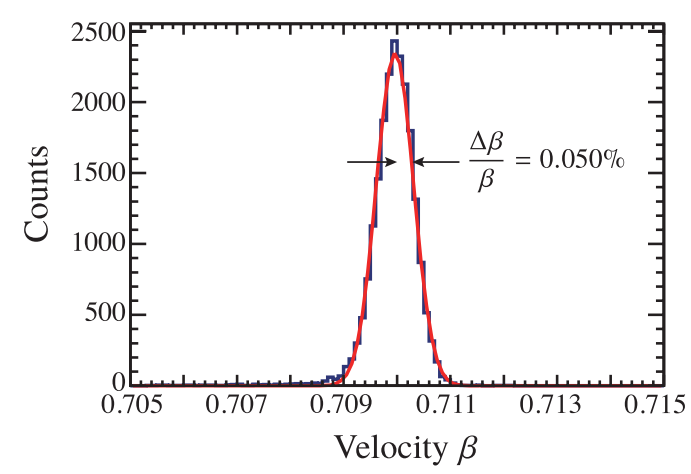

Figure 7: Velocity spectrum with a radiator $\left(\mathrm{SiO}_{2}\right)$ thickness of $0.95 \mathrm{~mm}$

Figure 8 shows a comparison of multiplicity dependence of the velocity resolution between the simulation and experimental results. Multiplicity is defined as the number of hit channels in an event. A red line and black points are the simulation and experimental data respectively. A blue histogram is an experimental multiplicity spectrum. As for experimental data points, some events were selected by multiplicity gates of each point. The simulation well reproduces the experimental data.

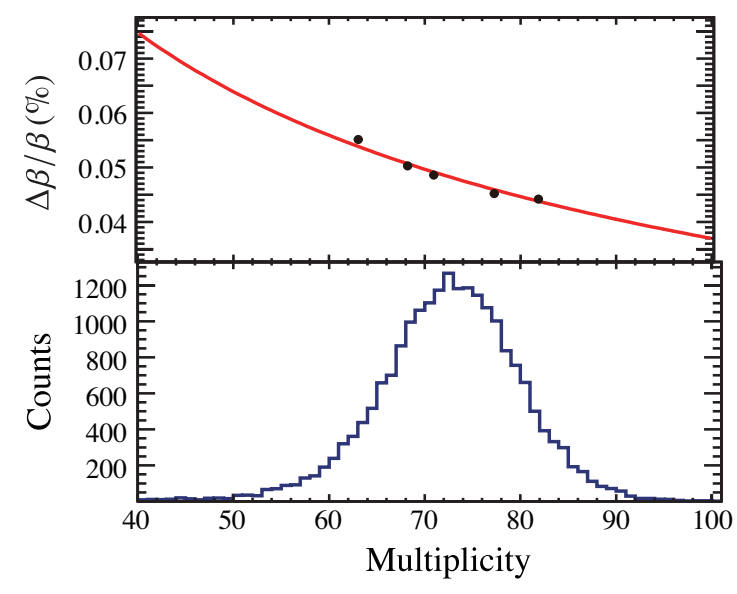

Figure 8: Multiplicity dependence of the velocity resolution

With the $0.95 \mathrm{~mm}$-thick radiator, a velocity resolution of $0.050 \%$ and a detection efficiency of $99.9 \%$ were achieved with $\beta=0.71$ and an atomic number $Z=54$. This is equivalent to TOF resolution at a distance of $60 \mathrm{~m}$ with two detectors of $\sigma=100 \mathrm{ps}$ which is a typical time resolution of plastic scintillators.

\subsection{Secondary beam}

In the secondary beam, the target material was a $2 \mathrm{~mm}$-thick beryllium and the radiator was BK7. A particle identification was successfully performed with HI-RICH and the ion chamber which is an energy loss $\Delta E$ detector. Figure $Q$ shows PID plots of the atomic number $Z$ and the velocity $\beta . Z$ was deduced by the ion chamber and HI-RICH. As can be seen, nuclides are separated 
clearly. In terms of ${ }^{113} \mathrm{Sn}$ as a typical example, a velocity resolution of $0.086(1) \%$ was achieved which is equivalent to $5.3 \sigma$ separation about the mass number as shown in Fig. 10 . All detection efficiencies were $100 \%$.

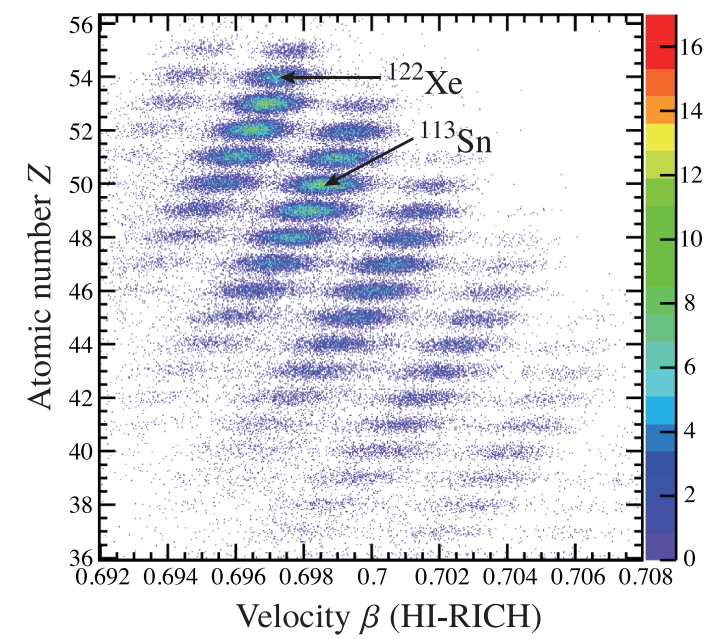

Figure 9: PID plots of the atomic number and the velocity

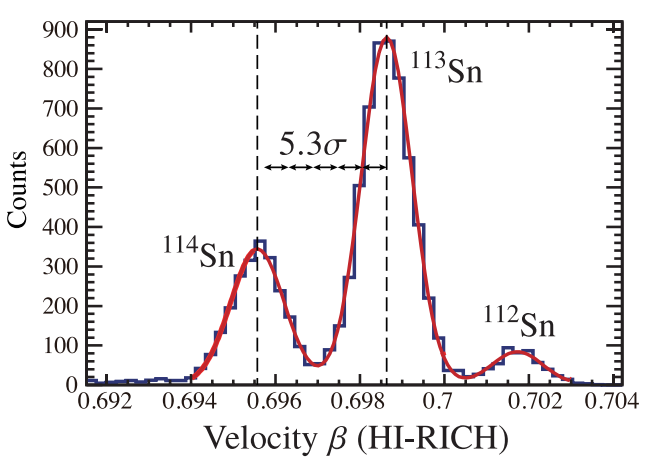

Figure 10: Projection of PID plots at $\mathrm{Sn}^{50+}$

Figure $\square$ shows a relation between the number of photons which is estimated by the experimental multiplicity and $Z^{2} \sin ^{2} \theta_{\mathrm{C}}$ which is a term of Eq. (ㅍ.L). These nuclides are selected from the line including ${ }^{113} \mathrm{Sn}$. According to Eq. (ㅁ. $\mathbb{C}$ ), the number of photons is theoretically proportional to $Z^{2} \sin ^{2} \theta_{\mathrm{C}}$, so that it can be said that the result is consistent.

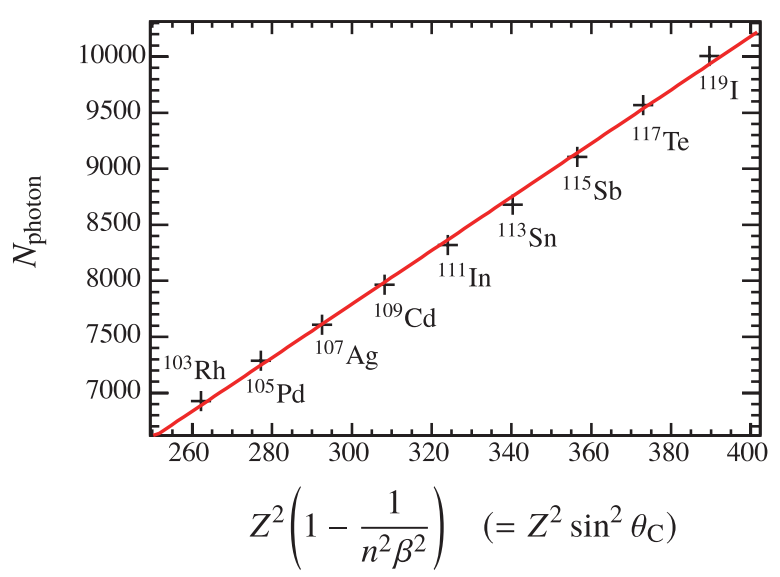

Figure 11: Relation between the number of photons and $Z^{2} \sin ^{2} \theta_{C}$ which is a term of Eq. (ㅁ.

\section{Summary}

We have developed a RICH for heavy ions with several hundreds MeV/u energy. The experiment was performed at NIRS-HIMAC. HI-RICH is consist of a radiator and three pairs of 
$8 \times 8$ multi-anode photomultiplier tubes which are covered with band-pass filters to suppress optical dispersion. The nucleus of the primary beam was ${ }^{132} \mathrm{Xe}$ with an energy of $420 \mathrm{MeV} / \mathrm{u}$. In the secondary beam experiment, the target material was a beryllium with a thickness of $2 \mathrm{~mm}$.

As for the primary beam, a velocity resolution $\Delta \beta / \beta=0.050 \%(\sigma)$ and a detection efficiency $\varepsilon=99.9 \%$ were achieved. In regards to the secondary beam, a particle identification was successfully performed. In terms of typical nucleus ${ }^{113} \mathrm{Sn}, 5.3 \sigma$ separation about the mass number was achieved.

\section{References}

[1] J. Seguinot; T. Ypsilantis. Nucl. Instrum. and Meth. 142 (1977) 377-391.

[2] S. Iwata, et al. Prog. Theor. Exp. Phys. 2016, no.3, 033 H01.

[3] R. Gemhauser, et al. Nucl. Instrum. and Meth. A. 433 (1999) 217-221.

[4] M. Kanazawa, et al. Nucl. Phys. A 746 (2004) 393-396

[5] Edmund Optics. "U-360 UV Bandpass Filter Coating Performance".

http://www. edmundoptics.com/document/download/353912 